\title{
GLOSSARY
}

\section{Alpha and Beta errors}

Alpha (Type I) errors are false-positives, ie, the results suggest that a treatment works when in fact it does not work. Beta (Type II) errors are false-negatives: results suggest a treatment does not work when in fact it does.

\section{Association}

A known link, or statistical dependence, between two or more conditions or variables, eg, statistics demonstrate that there is an association between smoking and lung cancer.

\section{Bias}

Something that introduces a difference or trend that distorts (or could distort) results of a study.

\section{Case-control study}

Compares people who have a disease or condition (cases) to another group of people from the same population who do not have that disease or condition (controls). A case-control study can identify risks and trends, and can suggest possible causes for disease or for particular outcomes.

\section{Case-series}

A report on a series of patients with an outcome of interest. No control group is involved.

\section{Cohort (study)}

A cohort is a clearly identified group of people: a cohort study follows that group over time and reports on what happens to them. A cohort study is an observational study and can be prospective or retrospective.

\section{Confidence interval}

Confidence interval (CI) is the range within which the true size of effect (never exactly known) lies with a given degree of assurance. People often speak of a $95 \% \mathrm{CI}$ (or 95\% confidence limits), the interval that includes the true value in $95 \%$ of cases.

\section{Confounding variable}

A variable that is not the one you are interested in but which may effect the results of the trial.

\section{Cross-over trial}

A trial where each of the groups will receive each of the treatments but in a randomised order, ie, they will start off in one arm of the trial but will deliberately cross over to the other $\operatorname{arm}(s)$ in turn.

\section{Cross-sectional study}

Also called a prevalence study: an observational study. It is analogous to taking a photograph of a group of people at one point in time and seeing the prevalence of diseases, for eg, in that population.

\section{Ecological survey}

A study based on aggregated data for some population as it exists at some point in time, to investigate the relationship of an exposure to a known or presumed risk factor for a specific outcome.

\section{Effectiveness (clinical effectiveness)}

The extent to which an intervention does people more good than harm. An effective treatment or intervention is effective in real-life circumstances, not just an ideal situation.

\section{Efficacy}

The extent to which an intervention improves the outcome for people under ideal circumstances. Testing efficacy means finding out whether something is capable of causing an effect at all.

\section{Heterogeneous (-geneity)}

The opposite of homogeneous. If a set of studies on the same subject have varied or conflicting results, the results of the group of studies are heterogeneous. Examining and explaining this heterogeneity is an important part of reviewing the research on a particular subject.

\section{Meta-analysis}

Meta-analysis is a statistical technique able to summarise the results of several studies into a single estimate, giving more weight to results from larger studies.

\section{Number-needed-to-treat (NNT)}

One measure of a treatment's clinical effectiveness. It is the number of people you would need to treat with a specific intervention (eg, aspirin for people having a heart attack) to see one occurrence of a specific outcome (eg, prevention of death).

\section{Randomised controlled trial}

A randomised controlled trial (RCT) is one in which subjects are randomly assigned to two groups, one (the experimental group) receiving the intervention that is being tested, and the other (the comparison group or controls) receiving an alternative treatment. The two groups are then followed up to see if any differences between them result. This allows assessment of the effectiveness of an intervention.

\section{Standard deviation}

The measure of how far data vary from the average (mean) result. The mean is the central (average) result. The standard deviation describes how far a value lies from the centre (mean, or average) value. A mean waiting time in a hospital emergency room may be $2 \mathrm{~h}$, but to cover most people's waiting time you might have to give or take an hour; the waiting time is therefore $2 \mathrm{~h} \pm 1 \mathrm{~h}$. That extra $1 \mathrm{~h}$ is the standard deviation. A person who waited $4 \mathrm{~h}$ to be seen would therefore be 2 standard deviations from the mean

\section{Statistical significance}

The findings of a study may be merely a fluke. A statistical test can determine whether or not the results of the study are likely to be a fluke or not. That test calculates the probability of the result being caused by chance.

\section{Validity}

The soundness or rigour of a study. A study is valid if the way it is designed and carried out means that the results are unbiased, ie, whether it gives you a true estimate of clinical effectiveness.

Note: some of the definitions are derived from those provided at http://www.cochrane.org/consumers/homepage.htm. 